\title{
The Emerging Role of Heme Oxygenase and Its Metabolites in the Regulation of Cardiovascular Function
}

\author{
David E. Stec, ${ }^{1}$ Kazunobu Ishikawa, ${ }^{2}$ David Sacerdoti, ${ }^{3}$ and Nader G. Abraham ${ }^{4}$ \\ ${ }^{1}$ Department of Physiology and Biophysics, Center for Excellence in Cardiovascular-Renal Research, \\ The University of Mississippi Medical Center, 2500 North State Street, Jackson, MS 39216, USA \\ ${ }^{2}$ Department of Cardiology, Center for Medical Education and Career Development, Fukushima Medical University, \\ 1 Hikarigaoka, Fukushima, Fukushima City 960-1295, Japan \\ ${ }^{3}$ Department of Clinical and Experimental Medicine, Clinica Medica 5, University of Padova, Via Giustiniani 2, 35100 Padova, Italy \\ ${ }^{4}$ Department of Physiology and Pharmacology, The University of Toledo College of Medicine, Toledo, OH 43614, USA
}

Correspondence should be addressed to David E.Stec, dstec@umc.edu

Received 18 July 2012; Accepted 18 July 2012

Copyright (C) 2012 David E. Stec et al. This is an open access article distributed under the Creative Commons Attribution License, which permits unrestricted use, distribution, and reproduction in any medium, provided the original work is properly cited.

Heme oxygenase $(\mathrm{HO})$ is an essential enzyme for normal homeostasis of the cardiovascular system. It is the enzyme responsible for the breakdown of heme to iron, biliverdin, and carbon monoxide (CO) gas. Biliverdin is then subsequently reduced to bilirubin by the ubiquitous enzyme biliverdin reductase. $\mathrm{HO}$ enzymes are essential for the turnover of red blood cells as well as of heme containing proteins within the cell. However, in recent years numerous studies have demonstrated an important role for $\mathrm{HO}$ and its metabolites, $\mathrm{CO}$, and bilirubin in regulating various physiological processes throughout the cardiovascular system. More importantly, alterations in $\mathrm{HO}$ have been identified in numerous pathological conditions, and therapies which induce $\mathrm{HO}$ have been found to provide beneficial outcomes in numerous cardiovascular disease processes. This special issue consists of 9 original research articles, 4 review articles, and 1 clinical study; all of which highlight the important role that $\mathrm{HO}$ enzymes have in the regulation of the cardiovascular system.

The important role of $\mathrm{HO}$ in the regulation of kidney function and blood pressure is highlighted in studies by $\mathrm{F}$. Botros et al., S. Quadri et al., and E. Csongradi et al. HO-1 induction with hemin results in marked increases in renal blood flow and glomerular filtration rate (GFR) which are associated with increases in urine flow and sodium excretion. HO-1 induction with hemin also attenuates the decrease in renal blood flow to acute angiotensin II (Ang II) treatment. The important role of HO-1 to counteract the effects of Ang II is further demonstrated in studies in by E. Csongradi et al. in which blockade of intrarenal HO-1 increases blood pressure in Ang II-dependent hypertension through increases in intrarenal superoxide production. The important antioxidant actions of $\mathrm{HO}$ and its metabolites are also made evident in studies by E. George et al. who demonstrate that both HO-1 induction and treatment with the $\mathrm{HO}$ metabolites $\mathrm{CO}$ and bilirubin attenuate the increase in oxidant production in placental explants in response to hypoxia. HO induction as well as $\mathrm{CO}$ and bilirubin also attenuate hypoxia-induced sFlt production which has important implications for pregnancy-induced hypertension or preeclampsia. Studies by T. Kawakami et al. also highlight the important antioxidant role of $\mathrm{HO}$ and its metabolites. In these studies, HO-1 induction lowers oxidative stress levels which in turn improves vascular function, lowers blood pressure, and prevents renal injury in extracellular superoxide dismutase (EC-SOD, SOD3) knockout mice. The important role of $\mathrm{HO}$ in the modulation of oxidative stress in the differentiation of bone-marrowderived mesenchymal stem cells (MSCs) and its potential role in diseases such as diabetes, inflammation, osteoporosis, and hypertension are reviewed by L. Vanella et al. HO-1 regulates MSC differentiation process by shifting the balance of MSC 
differentiation in favor of the osteoblast lineage by decreasing oxidative stress and increasing osteogenic markers such as alkaline phosphatase and BMP-2.

The important role of $\mathrm{HO}$ in target organ injury is also highlighted in this special issue. K. Chandrashekar et al. demonstrate the important role of $\mathrm{HO}-1$ in preserving renal function and protecting the kidney against renal injury in a model of subpressor Ang II-induced kidney injury. M. Constantin et al. detail the therapeutic potential of $\mathrm{HO}$ and its metabolite $\mathrm{CO}$ in lung inflammation, acute lung injury, lung transplantation, and pulmonary hypertension.

$\mathrm{HO}$ enzymes play a critical role in the regulation of vascular function and the protection of the vasculature from injury. The important role of $\mathrm{HO}$ enzymes in this capacity as well as the potential targeting of vascular $\mathrm{HO}$ for therapeutics is reviewed by E. Marcantoni et al. The importance of vascular HO-1 in the suppression of vascular inflammation is demonstrated in studies by $\mathrm{K}$. Ishikawa et al. who show that HO-1 knockout mice exhibit enhanced vascular inflammation, atherosclerotic lesions, and increased oxidation of HDL.

$\mathrm{HO}$ enzymes have an important interaction with nitric oxide (NO) in the regulation of vasculature tone. $\mathrm{HO}$ in the vasculature has been previously reported to both induce as well as inhibit the formation of $\mathrm{NO}$ depending on the degree of HO-1 induction. A. Daiber et al. examine the complex relationship between $\mathrm{HO}$ and $\mathrm{NO}$ and its implications in organic nitrate therapy and argue for a beneficial role of HO-1 induction to protect against tolerance to effective organic nitrate-based therapies for cardiovascular disease. The relationship between $\mathrm{HO}$ and $\mathrm{NO}$ is further investigated in a clinical study by D. Sacerdoti et al. who demonstrate the important role for $\mathrm{HO}$ in the maintenance of blood flow in the face of NO inhibition as well as the important role for $\mathrm{HO}$ in protecting the bioavailability of vascular-derived $\mathrm{NO}$ in humans.

The role of $\mathrm{HO}$ and its metabolites in metabolic diseases such as diabetes and obesity is also highlighted in this special issue. A. Elmarakby et al. report that induction of HO-1 is able to lower renal oxidative stress, inflammation, and injury in diabetic spontaneously hypertensive rats (SHRs) suggesting that induction of $\mathrm{HO}-1$ may be a potential therapeutic approach for diabetic nephropathy. J. Cao et al. elegantly demonstrate that HO-1 induction with the Apo AI mimetic, L-4F, decreases blood pressure, insulin resistance, blood glucose, and adiposity in HO-2 knockout mice. These results suggest that $\mathrm{HO}-1$ induction could be a viable therapeutic approach for the correction of both metabolic and cardiovascular disorders in obesity.

Taken together, the series of articles in this special issue highlight the important regulatory role of $\mathrm{HO}$ and its metabolites in the cardiovascular system. The articles also demonstrate the important role that $\mathrm{HO}$ enzymes play in protecting the cardiovascular system from diseases such as hypertension, atherosclerosis, kidney disease, diabetes, and obesity. They also shed light on the potential therapeutic role for HO-1 induction to combat these diseases. It is clear from these articles that the $\mathrm{HO}$ system is indeed emerging as both an important regulator of cardiovascular function as well as an attractive target for the development of effective therapeutics.

\section{Acknowledgments}

The Guest Editors would like to express their sincerest gratitude to the contributors of the articles for this special issue of the International Journal of Hypertension. They would also like to express their deepest appreciation for the external reviewers who assisted in the review process as well as the Editorial and Production teams at Hindawi Publishing Corporation for making this Special Issue possible.

David E. Stec Kazunobu Ishikawa David Sacerdoti Nader G. Abraham 


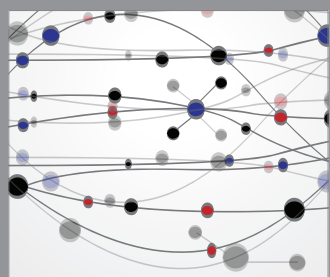

The Scientific World Journal
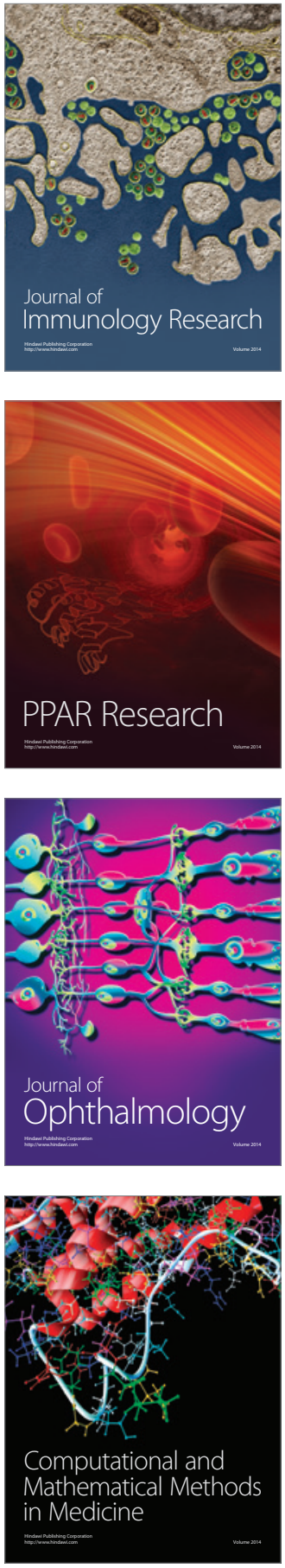

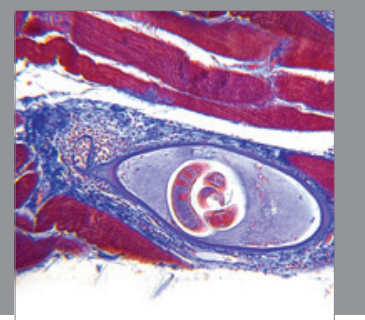

Gastroenterology

Research and Practice
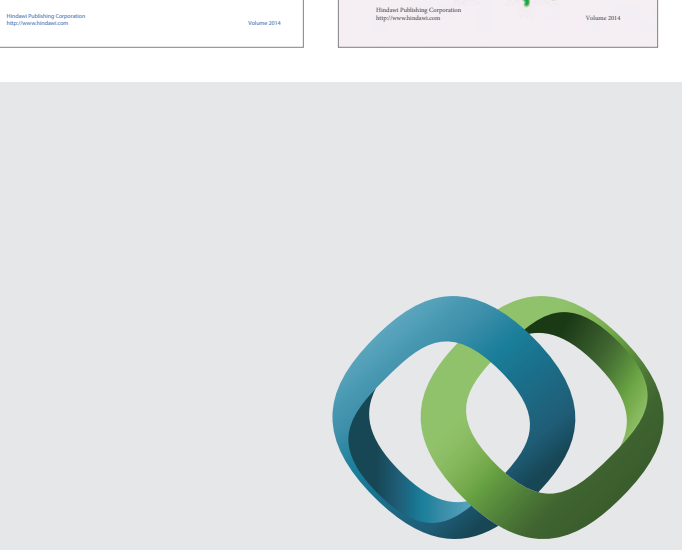

\section{Hindawi}

Submit your manuscripts at

http://www.hindawi.com
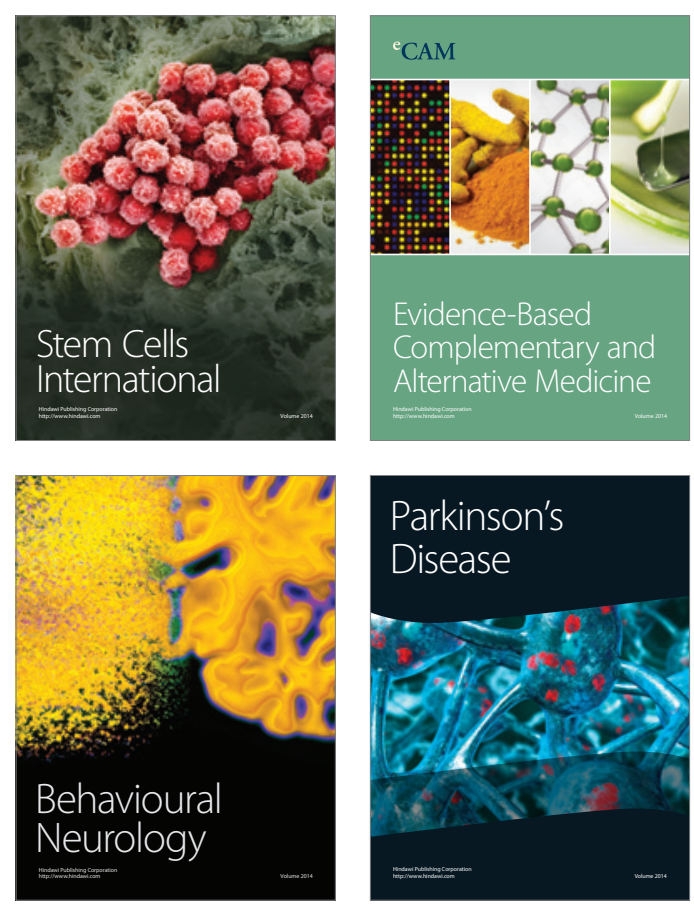

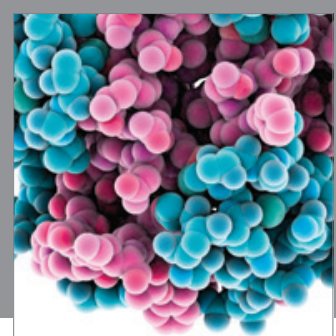

Journal of
Diabetes Research

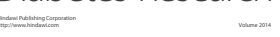

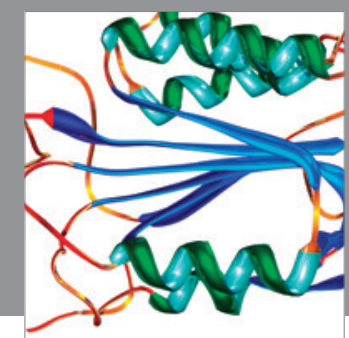

Disease Markers
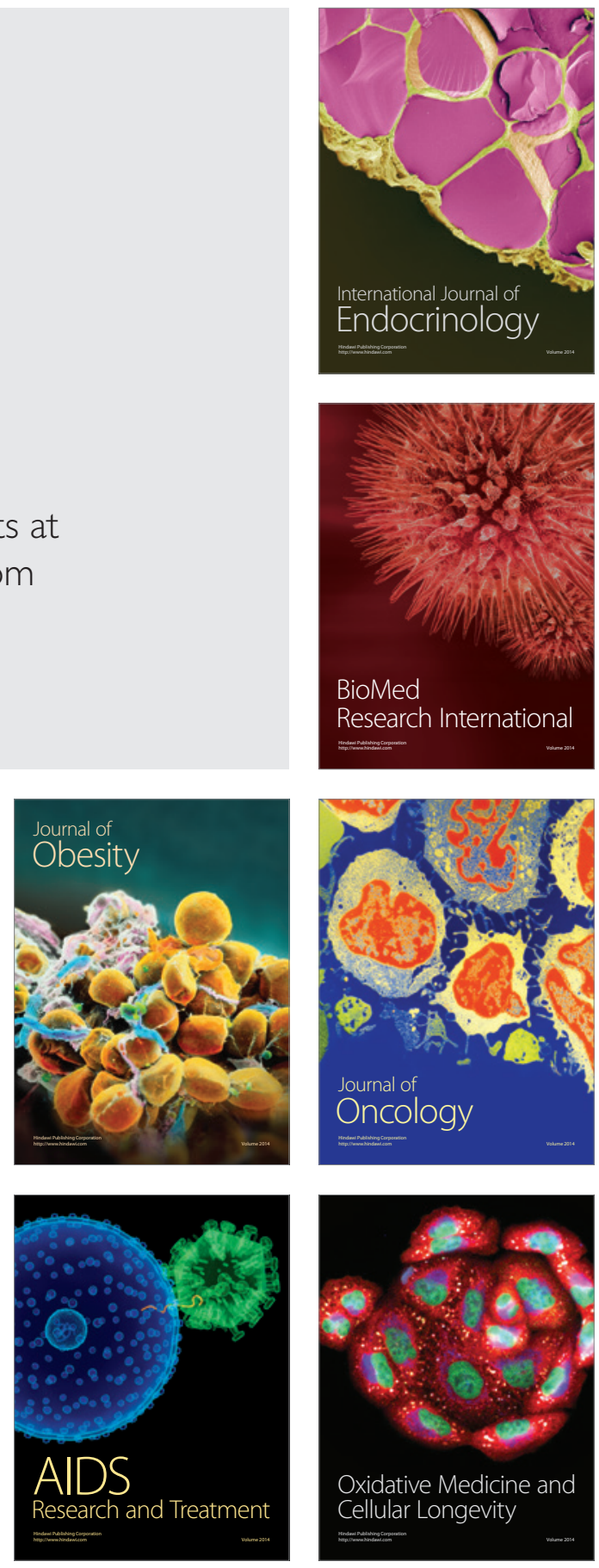